\title{
Direct measurement of molecular stiffness and damping in confined water layers
}

\author{
Steve Jeffery, ${ }^{1, *}$ Peter M. Hoffmann, ${ }^{2, \dagger}$ John B. Pethica, ${ }^{3}$ Chandra Ramanujan, ${ }^{1}$ H. Özgür Özer, ${ }^{3}$ and Ahmet Oral ${ }^{4}$ \\ ${ }^{1}$ Department of Materials, University of Oxford, Parks Road, Oxford OX1 3PH, United Kingdom \\ ${ }^{2}$ Department of Physics, Wayne State University, 666 W. Hancock, Detroit, Michigan 48201, USA \\ ${ }^{3}$ Department of Physics, Trinity College, Dublin, Ireland \\ ${ }^{4}$ Department of Physics, Bilkent University, Ankara, Turkey
}

(Received 9 October 2003; revised manuscript received 2 June 2004; published 31 August 2004)

\begin{abstract}
We present direct and linear measurements of the normal stiffness and damping of a confined, few molecule thick water layer. The measurements were obtained by use of a small amplitude $(0.36 \AA)$, off-resonance atomic force microscopy technique. We measured stiffness and damping oscillations revealing up to seven molecular layers separated by $2.526 \pm 0.482 \AA$. Relaxation times could also be calculated and were found to indicate a significant slow-down of the dynamics of the system as the confining separation was reduced. We found that the dynamics of the system is determined not only by the interfacial pressure, but more significantly by solvation effects which depend on the exact separation of tip and surface. The dynamic forces reflect the layering of the water molecules close to the mica surface and are enhanced when the tip-surface spacing is equivalent to an integer multiple of the size of the water molecules. We were able to model these results by starting from the simple assumption that the relaxation time depends linearly on the film stiffness.
\end{abstract}

DOI: 10.1103/PhysRevB.70.054114 PACS number(s): 68.08. - p, 07.79.Lh, 62.10.+s, 61.30.Hn

\section{INTRODUCTION}

The structure of water, as the primary biological solvent, has been intensively studied. For example, liquid water adopts short-range order, which depends strongly on dissolved species or geometric constraints. This structure emerges from the minimization of the free energy associated with the dynamic system of hydrogen bonds between neighboring water molecules. The entropy cost of the induced order almost certainly plays an important role in determining the structure of biological molecules that depend on hydration for their function, such as proteins and cell membranes. ${ }^{1}$

A surface can act as a model system for studying these phenomena, as it is both geometrically disrupting and can be chemically functionalized to affect the structure of the water close to it. A particularly interesting problem is the emergence of density oscillations as a function of film thickness when water is confined between two surfaces. ${ }^{2}$ This phenomenon is related to the radial density fluctuations in solvation shells of solutes. These density fluctuations have been originally observed by diffraction methods in clay-water systems. 3,4

In 1982, the mechanical response of confined water layers was directly determined using the surface force apparatus (SFA). ${ }^{5}$ With the invention of atomic force microscopy (AFM), attempts were made to measure stiffness oscillations with this technique. ${ }^{6}$ AFM probes have a much smaller contact area than SFA. This is an advantage if local changes in the water structure are to be examined, ${ }^{7}$ and potentially allows for probing regions of negative contact stiffness. The latter cannot be probed using SFA because the instrument stiffness is not high enough to withstand the snap-in instability in negative stiffness regions. The disadvantage of AFM is that the signals are much smaller and the contact area is determined by the tip shape and thus is essentially unknown. Also, the exact distance between the tip end and the surface is difficult to determine. The small signal-to-noise ratio in
AFM made the direct measurement of water structure an elusive goal. In 1995, Cleveland et al. ${ }^{8}$ measured the oscillatory potential of the confined water layers indirectly by analyzing the Brownian noise spectrum of a AFM tip immersed in water. More recently, direct measurements of the structure were achieved by Jarvis et al. ${ }^{9}$ by using nanotube probes and a large amplitude AFM technique, and by Antognozzi et al. ${ }^{10}$ who measured the local shear modulus using an AFM in shear force mode.

In this paper we present results of direct and linear measurements of the normal junction stiffness of water confined between the AFM tip and an atomically smooth mica surface. This was achieved by using ultrasmall amplitudes of $0.36 \AA$ and subresonance operation, which avoids the problem of reduced quality factor in liquids. The snap-in instability was avoided by using a sufficiently stiff cantilever (here $0.65 \mathrm{~N} / \mathrm{m}$ ). This method is ideal to make quantitative, point-by-point measurements of the mechanical properties of confined water layers. The linear measurements enabled us to reliably separate conservative and dissipative terms in the measurement for the first time. The small amplitudes (much smaller than the nominal size of a water molecule) allowed us to measure the elastic and viscous response of the confined water layer without disrupting the layers themselves, as would be the case in the large amplitude methods used previously. The challenge of such a technique is the measurement of exceedingly small signals, since the usual methods of signal enhancement (large amplitudes, low stiffness levers, resonance operation) are not used. Recently, we succeeded in implementing such a technique in UHV, ${ }^{11-13}$ and in liquids, ${ }^{14}$ using an improved fiber interferometric displacement sensor ${ }^{15}$ to overcome the reduced signal-to-noise ratio of the technique. Here we report on our direct measurements of the mechanical properties of confined water layers using this novel AFM technique. 


\section{EXPERIMENT}

Small amplitude, off-resonance $\mathrm{AFM}^{11}$ has recently been successfully used for measuring atomic bonding curves, ${ }^{12}$ mapping force gradients at atomic resolution, ${ }^{13}$ and measuring atomic scale energy dissipation. ${ }^{16}$ Both the force gradient and the damping coefficient/energy dissipation can be obtained by solving the equation of motion for a forced damped oscillator at a drive amplitude $A_{0} \ll \lambda$ (where $\lambda$ is the nominal range of the measured interaction) and $\omega \ll \omega_{0}$. The equation of motion is given by

$$
m \ddot{x}+\gamma \dot{x}+\left(k_{L}+k\right) x=k_{L} A_{0} \exp (i \omega t)
$$

where we linearized the force field, owing to the fact that lever amplitudes are much smaller than the range of the measured interactions, $\lambda$. This assumption has recently been shown to be justified if $A_{0}$ is sufficiently small. ${ }^{17}$ After solving the equation, we find for the interaction stiffness and the damping coefficient:

$$
k=k_{L}\left(\frac{A_{0}}{A} \cos \phi-1\right)
$$

and

$$
\gamma=-\frac{k_{L} A_{0}}{A \omega} \sin \phi
$$

Here, $A_{0}$ is the drive amplitude of the lever, $A$ is the measured amplitude as the surface is approached, $k_{L}$ is the lever stiffness, $\phi$ is the measured cantilever phase, and $k$ is the measured interaction stiffness. In Eq. (3), $\omega$ is the drive frequency and $\gamma$ is the damping coefficient. In performing the above calculations, we intrinsically assume that elastic and viscous forces are additive, i.e., they act in parallel (Kelvin or Voigt model). In modeling liquids, however, a Maxwell model tends to be more useful in which the elastic and viscous (damping) term are considered to be in series. Note that in general a more complicated combination of Maxwell and Kelvin elements should be used, which can represent multiple processes occurring at different time scales. In the measurements presented here we were somewhat limited to a narrow frequency range, since we had to keep the frequency below the cantilever resonance in order to ensure linearity. Thus we restricted ourselves to models with a single relaxation time, and were probing only processes with time scales comparable to the frequency of the vibrating cantilever. To convert from the Kelvin to the Maxwell model we can use the following set of equations: ${ }^{18}$

$$
\begin{gathered}
\eta=\gamma+\frac{k^{2}}{\omega^{2} \gamma}, \\
R=k+\frac{\omega^{2} \gamma^{2}}{k},
\end{gathered}
$$

where $\eta$ and $R$ are the viscous and elastic terms in the Maxwell model, respectively. This shows that mathematically the two models are equivalent since we can easily convert parameters from one model to the other. However, for finite values of $R$ and $\eta$ the Maxwell model allows for permanent strain without elastic recovery, while the Kelvin model always exhibits elastic recovery for finite values of $k$ and $\gamma$. Thus as far as the simplest models go, the Maxwell model is better suited to describe liquids than the Kelvin model.

The used AFM was homebuilt and incorporated a fiber interferometer mounted on a remote controlled nanomanipulator with five degrees of freedom and a step size of $<100 \mathrm{~nm}$. The nominal sensitivity of the interferometer was $3 \times 10^{-4} \AA / \sqrt{\mathrm{Hz}}$. For the results presented here, this allowed us to measure interaction stiffnesses of a few $10^{-2} \mathrm{~N} / \mathrm{m}$ using a $0.65 \mathrm{~N} / \mathrm{m}$ cantilever, a sub-angstrom lever amplitude of $0.36 \AA$, and reasonable integration times. The measurement frequency was $411 \mathrm{~Hz}$. The interferometer was also used to precisely calibrate the scanner piezo. Measurements were performed in ultrapure water with a concentration of $0.01 \mathrm{M} \mathrm{KCl}$. The surface was freshly cleaved mica, and the cantilever tip was made out of silicon. We performed measurements with several mica samples and silicon tips, as well as different amplitudes and (slightly) different frequencies. The results of all these measurements were consistent and exhibited the features discussed in this paper.

The tip shape was essentially unknown. It should be noted that it is extremely difficult to characterize the threedimensional shape of the tip at the required resolution of about $1 \AA$. In the future, more attention needs to be paid to the tip structure, and ways need to be found to characterize the tip before and after the measurement on as small a scale as possible. Despite these obvious limitations, we were able to take the tip shape into account by assuming a generic shape for the tip and found good agreement with experimental measurements. More details can be found towards the end of the paper.

It should also be noted that the absolute tip-surface separation is not known in AFM. Consequently, while individual water layers can be observed, it is not known how many total water layers are actually present in the gap.

\section{RESULTS}

Figure 1 shows the amplitude and the cantilever phase as a function of displacement. Displacement is measured from an (arbitrary) starting position and should not be confused with tip-sample separation. The surface is located to the right of the graph, and the monotonic drop-off of the amplitude as the surface is approached can be attributed to repulsive interactions. The monotonic ground is most likely due to hydrophilic forces, which dominate over the also present double-layer and van-der Waals forces. Repulsive background forces have also been observed in previous measurements by other groups. ${ }^{5}$

The amplitude data shows at least five equally spaced local minima (and maxima). The phase data shows equally spaced maxima further away from the surface, which roughly line up with the minima of the amplitude data. However, as the surface is further approached additional "intermediate" peaks appear close to the amplitude maxima, and these peaks finally dominate as the gap is decreased to a few molecular spacings. Overall, the phase increases up to a glo- 


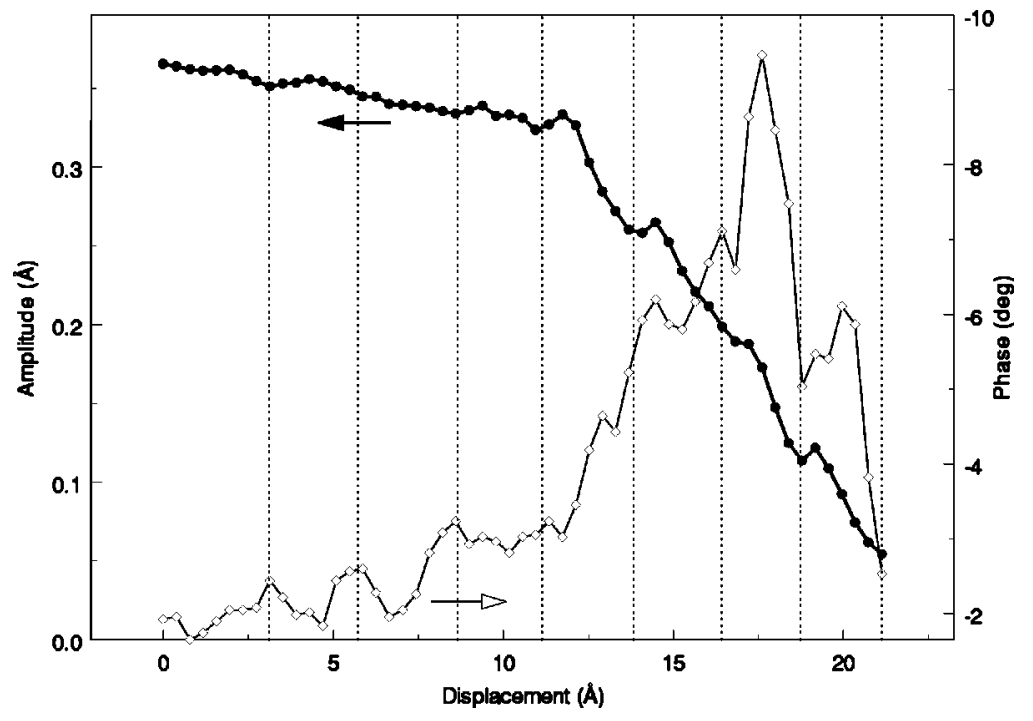

FIG. 1. Amplitude and phase measured on a water layer confined between the AFM tip and a mica surface. The mica surface is located to the right. Several oscillations can be seen in the amplitude data. The overall decrease as the surface is approached is due to hydrophilic effects. The phase shows a more complicated behavior (discussed in text), but also shows clear oscillations. The reference lines correspond to displacements where liquid ordering is maximized and serve as a guide for the eye.

bal maximum as the surface is approached and then decreases again closer to the surface.

The measured stiffness [Eq. (2)] can be decomposed into two components: A monotonic background, and an oscillatory term, which is the one we will be most concerned with in this paper. The monotonic background is most likely due to double-layer (DVLO) and hydrophilic interactions, which can both be modeled as exponentials.

As mentioned above we performed several measurements with different tips and amplitudes. All showed essentially the same features that are described in this paper. However, it should be noted that different tips give different background terms and different magnitudes of the stiffness and phase oscillations. Thus they cannot be simply averaged. Indeed, in our measurements we were able to see the stiffness oscillations in each measurement clearly without need of averaging. In Fig. 2 we present a set of measurements obtained with the same tip and at the same amplitude of $0.36 \AA$, as well as a composite model of the data based on all the measurements. The graphs in Fig. 2 were generated by a best fit and subsequent subtraction of the monotonic background, leaving only the stiffness oscillations associated with the layering of the confined water film. Note that the peaks in the stiffness data correspond to the minima in the amplitude data and thus to the higher stiffness of the ordered phase of the confined water layer. The specific data set shown in Fig. 1 is denoted by " 1 " in Fig. 2. In the subsequent discussion we will focus on this data set.

Based on the measurements in Fig. 2, the average spacing between the amplitude minima (and the phase maxima further out) is $2.523 \pm 0.340 \AA$, consistent with earlier reports. ${ }^{5,7-10}$ Overall, based on all measurements (with different tips, samples, and amplitudes; total number of measured oscillations $=30$ ) we found an average spacing of $2.526 \pm 0.482 \AA$.

Figure 3 shows the stiffness and damping coefficient calculated using Eq. (3) for the same data presented in Fig. 1 (data set "1" in Fig. 2). Close to the surface, the damping curve shows peaks that are "out of phase" with the stiffness data. Further away from the surface, however, double peaks occur, and finally the damping shows peaks that are in-phase with the stiffness maxima, similar to the phase data shown in Fig. 1. Due to the dissipation, the cantilever loses kinetic energy. The energy loss per cycle can be calculated from ${ }^{19}$

$$
E_{\mathrm{diss}}=\oint_{\text {cycle }} \gamma \dot{x} d x=\pi \gamma \omega A^{2}=\pi k_{L} A_{0} A \sin \phi .
$$

The maximum loss was $E_{\mathrm{diss}}=1.3 \mathrm{meV}$ per cycle, which was observed close to the maximum in the phase (Fig. 1).

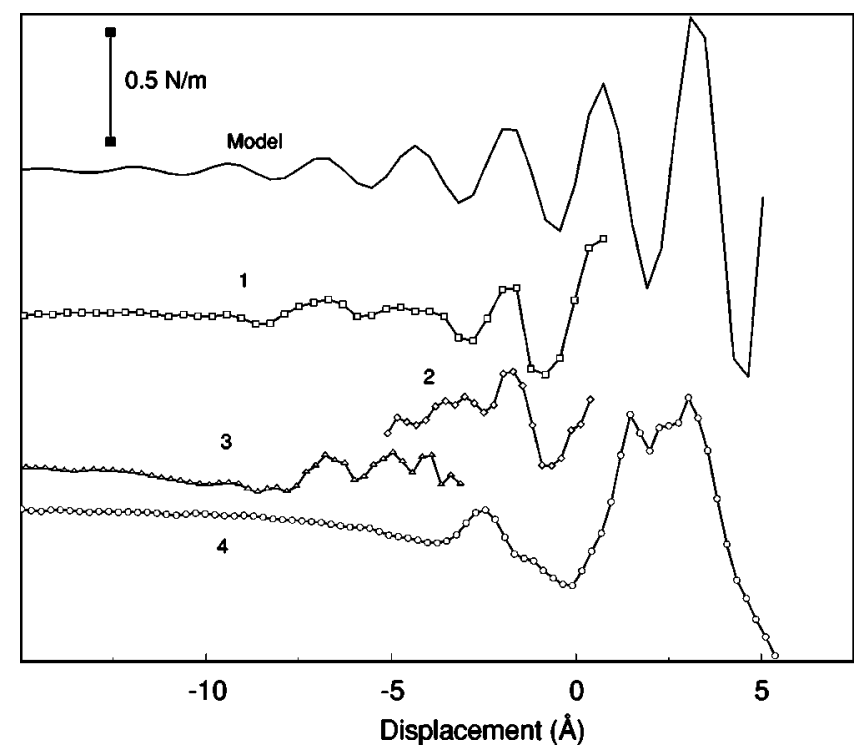

FIG. 2. Solvation stiffness versus displacement for four different measurements obtained with the same tip but at different times/ experiments, and at a lever amplitude of $0.36 \AA$. The solvation stiffness was obtained by calculating the stiffness from Eq. (2) and subtracting the exponential background. Differences in the curves can be attributed to noise, mechanical drift from the lever and piezo element, surface inhomogeneities, as well as changes in the tip structure after each measurement. Measurement " 1 " is analyzed in this paper (Figs. 1 and 3-5). A plausible composite of the measurements can be constructed, which is shown by the solid line (top) with a period of the stiffness oscillations of $2.523 \AA$, given by the average obtained from all four measurements. 


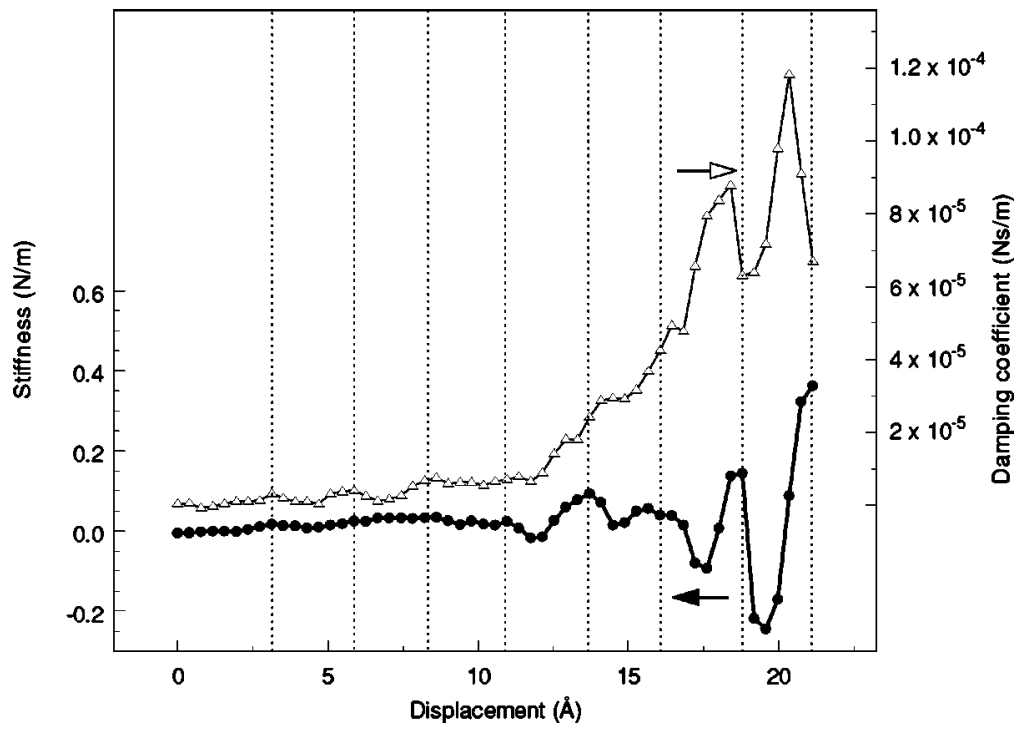

FIG. 3. Solvation stiffness and (Kelvin) damping coefficient versus displacement for measurement "1" in Fig. 2. The solvation stiffness was obtained by calculating the stiffness from Eq. (2) and subtracting the exponential background. In this figure, clear oscillations spaced at about $2.56 \AA$ can be seen in both the stiffness and the damping. The damping exhibits a "phase-shift" with respect to the stiffness data at a displacement of $15 \AA$. Closer to the surface the damping is out-of-phase with the stiffness, while further away it switches to being in-phase.

What is the origin of the observed dissipation and its increase as the surface is approached? One interpretation would be to attribute the damping to viscous drag, especially due to the squeezing of the liquid between the tip and the substrate. ${ }^{20}$ The squeeze Reynolds number, $R e$, is given by

$$
R e=\frac{\rho_{W} z}{\eta_{W}} \frac{d z}{d t}
$$

where $\eta_{W}$ is the viscosity of water, $\rho_{W}$ is its density, and $z$ is the tip-surface distance. In our case, $z \approx 1 \mathrm{~nm}, d z / d t \approx \omega A$ $\approx 100 \mathrm{~nm} / \mathrm{s}$, and we obtain $R e \approx 10^{-10} \ll 1$. In the following we assume that the viscous damping due to the cantilever beam does not change much with separation (since the beam is several micrometers away), and thus the variation of the damping with separation is dominated by the damping at the tip. With $R e \ll 1$, the squeeze damping term between tip and surface is given by ${ }^{20}$

$$
\gamma_{s}=6 \pi \eta_{W} \frac{R_{\mathrm{tip}}^{2}}{z} .
$$

Using reasonable values $(10-100 \mathrm{~nm})$ for the tip radius, $R_{\text {tip }}$, we find that the expected viscous damping at less than $1 \mathrm{~nm}$ separation is of the order of $10^{-9}$ to $10^{-7} \mathrm{Ns} / \mathrm{m}$, which is about three to four orders of magnitude smaller than the measured values (see Fig. 3), which are of order $10^{-5}$ to $10^{-4} \mathrm{Ns} / \mathrm{m}$. We found that the viscosity increased exponentially with distance and thus is large only very close to the surface $(<1 \mathrm{~nm})$. This effect has been observed before and has been attributed to a sharp increase in the effective viscosity of confined water layers, ${ }^{10,21,22}$ a possible indication of the altered dynamical and structural properties of liquids under confinement. However, there are currently a number of conflicting measurements and the nature of confined water layers is not entirely known. For example, Raviv et $a l{ }^{23,24}$ recently observed viscosities close to the bulk value even at separations as small as $1 \mathrm{~nm}$. Their results were based on measurements of the snap-in instability close to the surface, while in our experiments the mechanical properties of the film were measured continuously without any me- chanical instabilities. Moreover, in our experiment we considered normal forces, while their results are based on shear measurements. How these measurements relate to each other and if there is a fundamental difference between the normal and the lateral dynamic behavior of water is an important question for future study.

It should also be noted that there seem to be fundamental differences between water, 10,21-24 and most other liquids. ${ }^{20,25}$ For example, there are some indications that water does not "solidify" like other liquids, but instead becomes a more and more viscous liquid under confinement. ${ }^{22}$ Moreover, even in liquids that are believed to "solidify," the nature of the transition to the solid state is still under dispute. The transition has been described as a type of first-order phase transformation from liquid to solid, ${ }^{25}$ or, alternatively, as a continuous transformation, not unlike a glass transition. ${ }^{26}$

If the liquid does indeed turn solid under certain confinement conditions, the more appropriate mechanical model would be a Kelvin-type model. However, if the liquid stays essentially liquid albeit with greatly enhanced viscosity (recent evidence for this comes from diffusion measurements ${ }^{27}$ ), a Maxwell-type model should be used. As in any "standard" analysis of AFM, we used a Kelvin-type model above. However, to elucidate the nature of the changes under confinement further, it is important to use a model that more properly applies to liquids. Using Eqs. (4) and (5) we transformed the measured stiffness and damping terms to the Maxwell model. We found that the stiffness remains almost unchanged between the two models (i.e., $k \approx R$ ), but as shown in Fig. 4, the viscous term changes dramatically. The Kelvin model damping term, $\gamma$, is out-of-phase with the stiffness oscillations close to the surface (Fig. 3), while the Maxwell model damping, $\eta$, is much larger and essentially inphase with the stiffness variations (Fig. 4). As mentioned above, further away from the surface, the Kelvin damping experiences a "phase shift" and becomes in-phase with the stiffness (similar to the phase data in Fig. 1). The Maxwell damping, on the other hand, remains in-phase throughout. More about this below. 


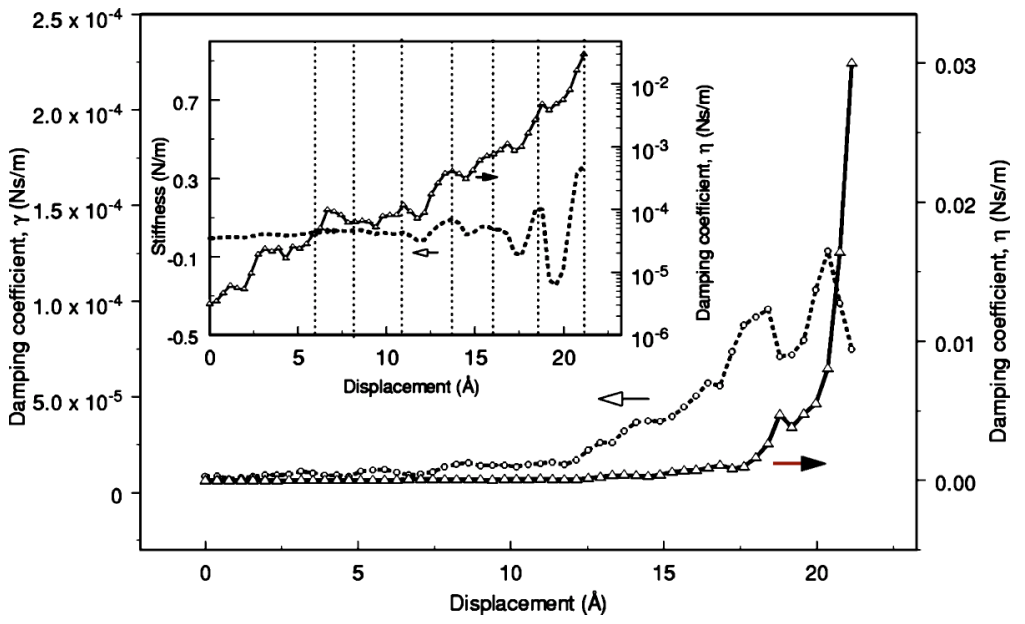

FIG. 4. Comparison of Kelvin damping, $\gamma$, and Maxwell damping, $\eta$. If we treat the confined film as a liquid (Maxwell-type model), we find that the damping increases very strongly as the film is squeezed to a few molecular layers, unlike the Kelvin damping which increases more moderately. The inset shows the Maxwell damping, $\eta$, on a log-scale, with the stiffness as a reference. It can be seen that $\eta$ is essentially in-phase with the stiffness throughout the measurement range.

When dealing with dissipative behavior it is useful to look at the characteristic time constants involved in the dynamic behavior of the confined liquid. In general, there will be a "spectrum" of such time constants corresponding to different processes occurring at different scales. As mentioned above, we limited ourselves to a single frequency measurement, and thus can explore only time constants that are of the order of the inverse of the cantilever frequency. In the Kelvin model, a characteristic time is given by $t_{c}=\gamma / k$, which is called the "retardation time." 18 This time is approximately the time needed to build up a significant strain in the material upon application of a constant stress. In standard solids, a certain amount of strain can be obtained almost instantaneously due to the elasticity of the material, however, in ideal liquids, instantaneous strain is not possible due to the velocitydependent damping. Thus a lower $t_{c}$ might indicate a more solidlike material. On the other hand, in the Maxwell model, the characteristic time is $t_{r}=\eta / R$, which is the "relaxation time." This time is related to the time needed for stresses in the material to relax after a strain has been imposed. In solids, stresses will persist for long times when a strain is applied (one of the characteristics of materials being solid), while in liquids any stresses will quickly dissipate away. Thus higher $t_{r}$ indicates a more solidlike behavior. It should be noted that $t_{c}$ and $t_{r}$ are simply related by

$$
t_{c}=\frac{1}{\omega^{2} t_{r}} .
$$

The dependence of $t_{c}$ and $t_{r}$ on displacement is shown in Fig. 5 . It can be seen that overall $t_{c}$ is decreasing and $t_{r}$ increasing exponentially as the liquid layer is increasingly confined. The relaxation time $t_{r}$ approaches $10^{-2} \mathrm{~s}$ close to the surface in good agreement with earlier reports based on shear measurements. ${ }^{21}$ This indicates a tendency for the layer to become more solidlike. Even more interesting, however, is the fact that at separations where the stiffness oscillations are at their maximum, $t_{c}$ is lowered and $t_{r}$ is increased. This is a further indication that the water becomes more solidlike when it is allowed to order, i.e., when the tip-surface separation is commensurate with the "natural" molecular spacing of water. In the "ordered phase," the stiffness $k$ (or $R$ ) is maximum, the retardation time, $t_{c}$, is minimum, and the relaxation time, $t_{r}$, is maximum, as expected for a solid.

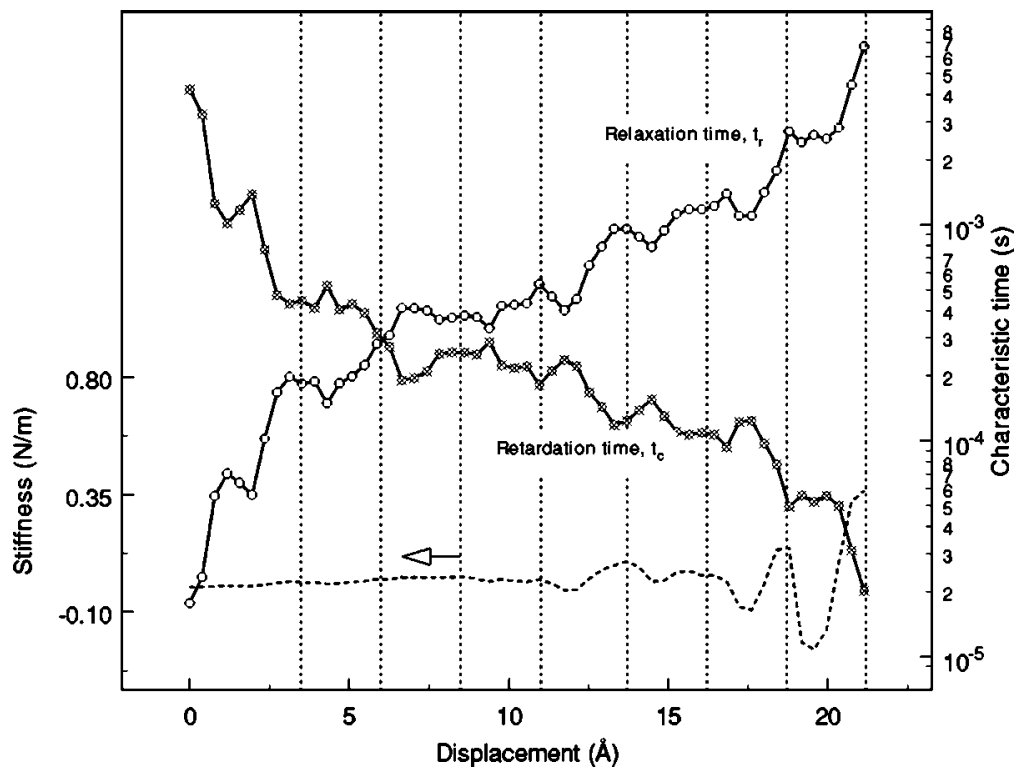

FIG. 5. Relaxation $\left(t_{r}\right)$ and retardation $\left(t_{c}\right)$ time plotted versus displacement. The relaxation time is in-phase with the stiffness, while the retardation time is out-of-phase. The relaxation time increases overall due to the increasing pressure, but also exhibits in-phase oscillations due to solvation effects. 
Another interesting observation is that the characteristic times $t_{r}$ and $t_{c}$ change very slowly with separation until a few angstroms from the surface. Then they seem to change more rapidly, with the liquid becoming seemingly even more solidlike. For nonaqueous liquids, some authors ${ }^{25}$ have argued that this behavior shows the liquid undergoes some kind of first order phase transformation upon confinement, while others suggest a more gradual, glasslike transition. ${ }^{26}$ As mentioned above, recent results measured under shear suggest that water in particular fails to "solidify" at all ${ }^{22}$ and might not even show a slow-down in its dynamics. ${ }^{23,24}$ Our results seem to suggest slower dynamics close to the surface and possibly a more significant change as the water layer is reduced to a few molecular layers. Moreover, as we will see below, there seems to be both a gradual stiffening of the layer as pressure is applied and a much more pronounced periodic change of the mechanical behavior of the confined film associated with layering.

\section{DISCUSSION AND MODEL}

There are several surprising findings from the linear measurement of confined water presented here: (1) observed oscillations in the phase and dissipation extend much further than the oscillations in the amplitude or stiffness; (2) the phase and the Kelvin damping oscillations experience a "phase shift" with respect to the stiffness data as we move away from the surface; (3) while on average the amplitude continuously decreases as the surface is approached, the phase seems to pass through an intermediate maximum, and, finally, as hinted above; (4) the mechanical behavior of the layer changes both gradually (hydrophilic background) and more abruptly (solvation shell oscillations). To explain this behavior we simulated the nanomechanical behavior of the water layer by starting from the assumption that the relaxation time, $t_{r}$, is to first order linearly dependent on the stiffness of the water layer. This is not to be taken literally, in the sense of a direct physical connection between the stiffness and the relaxation time (although there well might be), but rather the stiffness is seen as an indicator of the "solidness" of the layer, and the relaxation time (as another indicator) is taken to be essentially proportional to it. We found that we can get the best fit of our data if we assume that the relaxation time depends linearly on both the background stiffness, $k_{h}$, due to hydrophilic interaction and on the stiffness oscillations, $k_{s}$, due to the solvation effects but with two different "coupling" constants $\alpha_{1}$ and $\alpha_{2}$ :

$$
t_{r}=\alpha_{1} k_{h}+\alpha_{2} k_{s}+t_{0} .
$$

Here, $t_{0}$ corresponds to the relaxation time measured far away from the surface. The advantage of using separate constants $\alpha_{1}$ and $\alpha_{2}$ is that we can separate the effect of background hydrophilic interactions from the influence of solvation forces on the relaxation time. We found that in order to reproduce the experimental results as closely as possible it was necessary to set $\alpha_{1}$ to $2.3 \times 10^{-3} \mathrm{sm} / \mathrm{N}$ and $\alpha_{2}$ to $5 \times 10^{-3} \mathrm{sm} / \mathrm{N}$, i.e., the relaxation time was more than twice as sensitive to solvation forces than it was to the hydrophilic background. It should be noted that the hydrophilic back- ground stiffness is directly proportional to the load (or surface pressure), since both are exponentials and one is the derivative of the other. Thus by the above approach we can separate the effects of the overall pressure or load from the effect of the liquid ordering which only occurs at certain, molecularly commensurate separations.

The retardation time, $t_{c}$, can be calculated from Eq. (9). The damping coefficients $\gamma$ and $\eta$ are then given by

$$
\begin{aligned}
& \gamma=t_{c} \cdot k, \\
& \eta=t_{r} \cdot R .
\end{aligned}
$$

In the simulations, we took $k=R$ (as found experimentally) to simplify the calculations. The solvation force was modeled as follows: ${ }^{2}$

$$
F_{s}=\sum_{\text {tip }, k=0}^{N} 2 \pi r^{2}\left(z_{k}\right) \times k_{B} T \rho \cos \left(\frac{2 \pi\left(z_{k}+D\right)}{\sigma}\right) \exp \left(-\frac{z_{k}+D}{\sigma}\right)
$$

where we summed the contributions of different areas of the tip by subdividing the tip into $N$ horizontal "slices" of radius $r\left(z_{k}\right)$. Here, $\rho$ is the particle density of water, $\sigma$ is both the period and the decay parameter of the interaction (they were experimentally found to be nearly identical), $z_{k}$ is the height of the $k$ th slice of the tip, and $D$ is the tip-surface separation. The hydrophilic interaction is given by

$$
F_{h}=\sum_{\text {tip }, k=0}^{N} 2 \pi r^{2}\left(z_{k}\right) \cdot p_{h} \exp \left(-\frac{z_{k}+D}{\lambda}\right)
$$

where $p_{h}$ is a constant and $\lambda$ is the decay parameter of the hydrophilic background. All parameters in expressions (13) and (14) were determined from the experiment. The hydrophilic decay parameter was found to be only slightly smaller $(\lambda=2.45 \AA)$ than the decay parameter of the oscillations $(\sigma=2.56 \AA)$. The corresponding stiffnesses were found from taking the derivative of the forces with respect to tipsurface separation, $k=-d F / d D$.

Since we cannot know the exact geometry of the tip, we did not expect to get a perfect agreement between theory and experiment. Nevertheless we obtained a semiquantitative agreement that reproduces all of the surprising features mentioned above. The geometry of the tip was assumed to be paraboloid, and the best agreement with experimental data was obtained for a nominal radius of $1 \mathrm{~nm}$. Figure 6 shows the calculated total stiffness and Kelvin damping coefficient, $\gamma$. The damping coefficient is out-of-phase with the stiffness close to the surface, then undergoes a "phase shift" and becomes in-phase further from the surface, as seen in the experiment. From Eq. (11) we see that the damping is a product of the retardation time $t_{c}$ and the stiffness $k$. Close to the surface the damping is dominated by the retardation time $t_{c}$, which is always out-of-phase with the stiffness (Fig. 5), while further away from the surface the stiffness $k$ dominates the variation in the damping. On the other hand, the Maxwell damping, $\eta$, is always in phase with the stiffness, since the relaxation time $t_{r}$ is in-phase with $R$ (or $k$ ). 


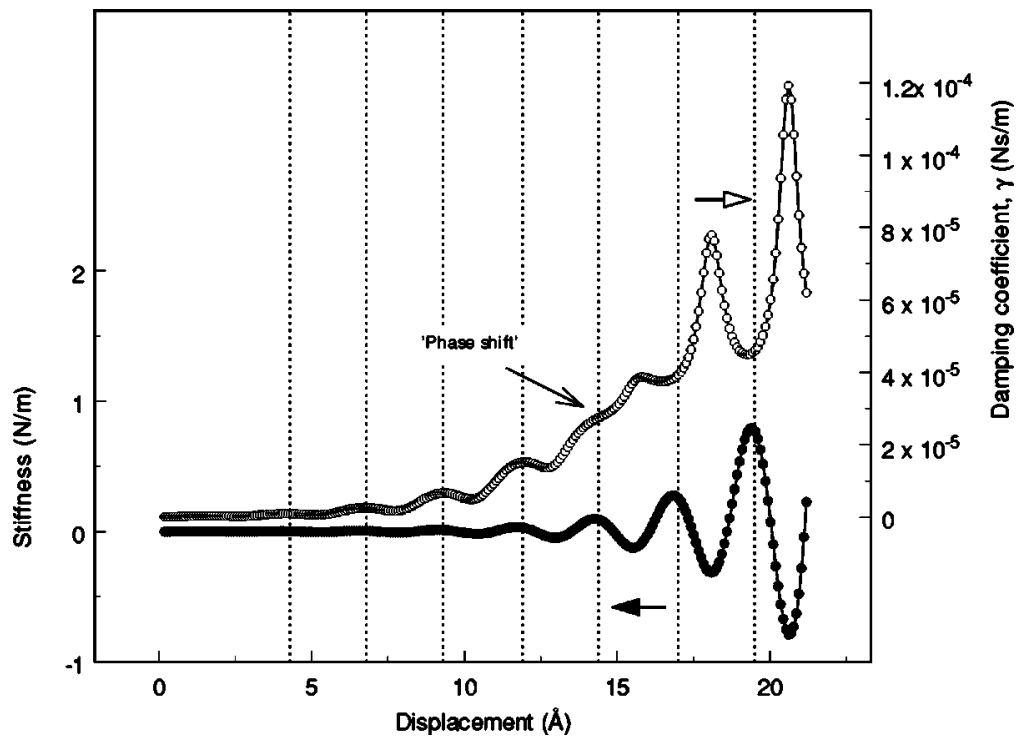

FIG. 6. Simulated stiffness and Kelvin damping, $\gamma$. Compare to measured data (Fig. 3). Although we had to assume larger stiffness oscillations in the model, the overall agreement is good, and the "phase shift" in the damping data is reproduced well.
It can be shown that a more complicated mechanical model, such as the commonly used Burger's model, behaves like a Maxwell model at low frequencies. This implies that the present discussion has more general implications than might be expected from the use of such simplified mechanical models. In particular, it would seem from Eq. (11) that the oscillatory behavior of the Kelvin damping, $\gamma$, could be explained by the oscillatory behavior of the stiffness, even if the retardation time is constant or slowly varying. In this scenario, the observed oscillations of the retardation/ relaxation time would be merely an "artifact" of the calculation. However, if the retardation time were constant or smoothly varying (i.e., not oscillating), the Kelvin damping would have to remain in-phase with the stiffness at all times. This is not observed in the experiment. The fact that $\gamma$ is out-of-phase close to the surface implies that the relaxation/ retardation time of the liquid exhibits separation-dependent oscillations independent of the oscillations of the stiffness. This means that the dynamic behavior of the confined liquid is strongly affected by how commensurate the tip-surface spacing is with regard to the size of the confined molecules.

The phase was calculated by solving Eqs. (2) and (3) simultaneously (and assuming $\omega \ll \omega_{0}$ ):

$$
\tan \phi=\frac{\omega \gamma}{k+k_{L}}
$$

The simulated phase, $\phi$, is shown in Fig. 7. The simulation reproduces all the "puzzling" features of the experiment: the shift from being out-of-phase to being in-phase with the stiffness oscillations and the intermediate maximum in the phase. The shift is due to the shift in $\gamma$ discussed above. The intermediate maximum is due to the fact that the stiffness changes slowly far from the surface, but then rather rapidly closer in, "overtaking" the damping coefficient in the process [Eq. (15)]. The observation that oscillations in the phase or damping are observable further away from the surface than the oscillations of the stiffness can also be explained: As we can

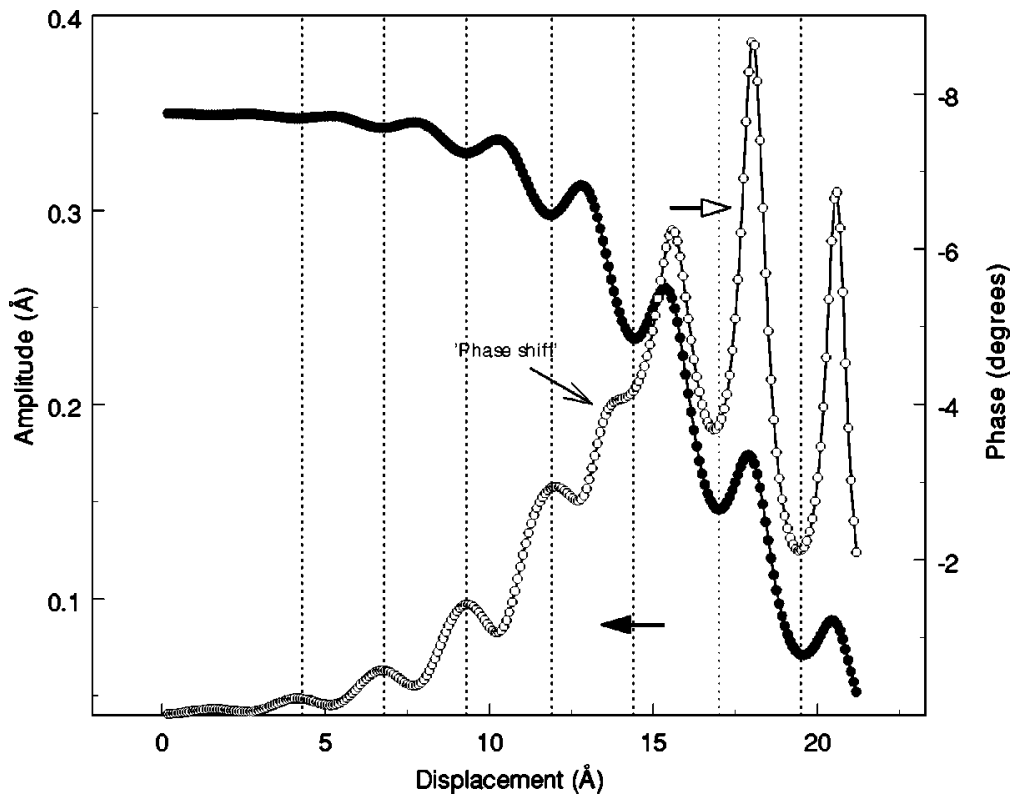

FIG. 7. Simulated amplitude and phase. Compare to measured data (Fig. 1). There is good qualitative agreement and the complicated behavior of the phase is well reproduced including the "phase shift" with respect to the stiffness (here: amplitude) and the global maximum. 
see in the simulated phase, a phase of more than $1^{\circ}$ is observed as far away as $13 \AA$ from the closest approach (about five water layers). Such a phase angle can be easily measured with a lock-in amplifier. On the other hand, at the same separation, the stiffness is only $0.04 \mathrm{~N} / \mathrm{m}$ requiring a measurement of a change in lever amplitude of the order of $0.02 \AA$, which is more difficult to measure and can be lost in the noise.

In conclusion, we can see that our simple approach of directly relating the relaxation time to the stiffness of the layer has allowed us to reproduce all the important features of the experiment. The relaxation/retardation times can therefore be taken as additional physical parameters (together with the stiffness) that characterize the mechanical properties of the system. The weaker dependence of the relaxation time on the hydrophilic (background) interaction and the more pronounced dependence on the oscillatory solvation forces suggests a compromise in the continuing debate over the nature of the solid-liquid transition. It seems that there is a gradual increase of the relaxation time with surface pressure and a more substantial change related to the molecular ordering of the liquid close to the surface.
*Present address: Oak Hill Theological College, Southgate, London N14 4PS, United Kingdom.

†Electronic address: hoffmann@physics.wayne.edu

${ }^{1}$ J. L. Finney, Faraday Discuss. Chem. Soc. 103, 1 (1996).

${ }^{2}$ J. N. Israelachvili, Intermolecular \& Surface Forces (Academic Press, London, 1992), Chap. 13.

${ }^{3}$ W. F. Bradley, R. E. Grim, and G. L. Clark, Z. Kristallogr. 97, 216 (1937).

${ }^{4}$ U. Del Pennino, E. Mazzega, S. Valeri, A. Aliette, M. F. Brigatti, and L. Poppe, J. Colloid Interface Sci. 84, 301 (1981).

${ }^{5}$ J. N. Israelachvili and R. M. Pashley, Nature (London) 306, 249 (1983).

${ }^{6}$ S. J. O'Shea, M. E. Welland, and T. Rayment, Appl. Phys. Lett. 60, 2356 (1992)

${ }^{7}$ D. L. Patrick and R. M. Lynden-Bell, Surf. Sci. 380, 224 (1997).

${ }^{8}$ J. P. Cleveland, T. E. Schäffer, and P. K. Hansma, Phys. Rev. B 52, R8692 (1995).

${ }^{9}$ S. P. Jarvis, T. Uchihashi, T. Ishida, H. Tokumoto, and Y. Nakayama, J. Phys. Chem. B 104, 6091 (2000).

${ }^{10}$ M. Antognozzi, A. D. L. Humphris, and M. J. Miles, Appl. Phys. Lett. 78, 300 (2001).

${ }^{11}$ A. Oral, R. A. Grimble, H. Ö. Özer, and J. B. Pethica, Rev. Sci. Instrum. 74, 3656 (2003).

${ }^{12}$ P. M. Hoffmann, A. Oral, R. A. Grimble, H. Ö. Özer, S. Jeffery, and J. B. Pethica, Proc. R. Soc. London, Ser. A 457, 1161 (2001).
${ }^{13}$ A. Oral, R. A. Grimble, H. Ö. Özer, P. M. Hoffmann, and J. B. Pethica, Appl. Phys. Lett. 79, 1915 (2001).

${ }^{14}$ P. M. Hoffmann, S. Jeffery, A. Oral, R. A. Grimble, H.Ö. Özer, and J. B. Pethica, Mater. Res. Soc. Symp. Proc. 649, Q9.2 (2001).

${ }^{15}$ D. Rugar, H. J. Mamin, and P. Guethner, Appl. Phys. Lett. 55, 2588 (1989).

${ }^{16}$ P. M. Hoffmann, S. Jeffery, J. B. Pethica, H. Ö. Özer, and A. Oral, Phys. Rev. Lett. 87, 265502 (2001).

${ }^{17}$ P. M. Hoffmann, Appl. Surf. Sci. 210, 140 (2003).

${ }^{18}$ W. N. Findley, J. S. Lai, and K. Onaran, Creep and Relaxation of Nonlinear Viscoelastic Materials (Dover, New York, 1976), Chap. 5.

${ }^{19}$ B. Anczykowski, B. Gotsmann, H. Fuchs, J. Cleveland, and V. B. Elings, Appl. Surf. Sci. 140, 376 (1999).

${ }^{20}$ S. J. O'Shea and M. E. Welland, Langmuir 14, 4168 (1998).

${ }^{21}$ A. Dhinojwala and S. Granick, J. Am. Chem. Soc. 119, 241 (1997).

${ }^{22}$ Y. Zhu and S. Granick, Phys. Rev. Lett. 87, 096104 (2001).

${ }^{23}$ U. Raviv, P. Laurat, and J. Klein, Nature (London) 413, 51 (2001).

${ }^{24}$ U. Raviv and J. Klein, Science 297, 1540 (2002).

${ }^{25}$ J. Klein and E. Kumacheva, J. Chem. Phys. 108, 6996 (1998).

${ }^{26}$ A. L. Demirel and S. Granick, Phys. Rev. Lett. 77, 2261 (1996).

${ }^{27}$ A. Mukhopadhyay, J. Zhao, S. C. Bae, and S. Granick, Phys. Rev. Lett. 89, 136103 (2002). 